\title{
Aldehyde dehydrogenase activity in cryopreserved cord blood cells for quality assessment prior to transplantation
}

\author{
JUNKO IKEMOTO $^{1}$, SATOSHI YOSHIHARA ${ }^{1}$, TAMAMI KOBAYASHI ${ }^{1,2}$, \\ SHUNRO KAI ${ }^{1,2}$ and YOSHIHIRO FUJIMORI ${ }^{1,2}$ \\ ${ }^{1}$ Center for Blood Transfusion and Cellular Therapy, Hyogo College of Medicine Hospital; \\ ${ }^{2}$ Hyogo Cord Blood Bank, Nishinomiya, Hyogo 663-8501, Japan
}

Received March 1, 2016; Accepted February 9, 2017

DOI: $10.3892 / \mathrm{mmr} .2018 .9481$

\begin{abstract}
In umbilical cord blood transplantation (UCBT), the number of cluster of differentiation (CD) $34^{+}$cells and colony-forming units (CFUs) in the cord blood (CB) graft positively correlate with patient survival. Therefore, these parameters are currently used for quality assessment of the cryopreserved $\mathrm{CB}$ cells in the attached segment that is considered representative of the $\mathrm{CB}$ in the main bag prior to UCBT. Since aldehyde dehydrogenase (ALDH) activity is high in hematopoietic stem cells, the number of ALDH-bright $\left(\mathrm{ALDH}{ }^{\mathrm{br}}\right)$ cells was examined in comparison with the number of $\mathrm{CD} 34^{+}$cells and CFUs for the quality assessment of $\mathrm{CB}$ units. In the cryopreserved main bag, the number of $\mathrm{ALDH}^{\text {br }}$ cells in the $\mathrm{CB}$ unit exhibited positive correlation with the number of $\mathrm{CD}_{3} 4^{+}$cells, and with CFU-granulocytes/macrophages and total CFU counts. Furthermore, the concentration of $\mathrm{ALDH}^{\mathrm{br}}$ cells in the cryopreserved attached segment was not significantly different compared to that of the main bag, suggesting that the attached segment is representative of the main bag. In conclusion, the present study suggested that $\mathrm{ALDH}^{\mathrm{br}}$ cell counts in the cryopreserved attached segments may serve as a quality assessment indicator for $\mathrm{CB}$ units prior to UCBT.
\end{abstract}

\section{Introduction}

Umbilical cord blood transplantation (UCBT) is increasingly performed as an alternative treatment for hematological malignancies, including leukemia and lymphomas $(1,2)$. Hematopoietic stem cells in cord blood (CB) are immature and limited in number, which may correlate to the relatively high incidence of engraftment failure and delays in UCBT (3). Hematopoietic stem

Correspondence to: Dr Yoshihiro Fujimori, Center for Blood Transfusion and Cellular Therapy, Hyogo College of Medicine Hospital, 1-1 Mukogawa-cho, Nishinomiya, Hyogo 663-8501, Japan E-mail: fuji-y@hyo-med.ac.jp

Key words: cord blood cells, cryopreservation, aldehyde dehydrogenase, colony forming units, cluster of differentiation 34 cell activity is routinely estimated by flow cytometric detection of cluster of differentiation (CD) $34^{+}$cells, and by colony-forming unit (CFU) assays in which the ability of blood cells to form colonies in a semisolid medium is tested, for example, formation of granulocyte/macrophage (GM) colonies in the CFU-GM assay. Predictive markers of CB engraftment are important for successful UCBT, and reports suggest that increased numbers of total nucleated cells (TNC), CD34 ${ }^{+}$cells (3), and CFU-GM or total CFUs (4) in the CB graft are associated with better engraftment and survival in UCBT.

$\mathrm{CB}$ for transplantation is currently provided by the $\mathrm{CB}$ bank (CBB), where $\mathrm{CB}$ units (CBUs) are cryopreserved following harvesting during full term delivery. As cryopreservation causes a decrease in the absolute number of TNCs and CD34 ${ }^{+}$ cells (5), a quality assessment following thawing is performed on selected CBUs (6). Because the cryopreserved CB in the main bag is shipped for transplantation, the $\mathrm{CB}$ in the attached segment provides the only source for indirect assessment of the CB quality in the man bag (7).

Aldehyde dehydrogenase (ALDH) is a cytosolic enzyme is responsible for the oxidation of intracellular aldehydes. In an ALDH activity detection system (ALDEFLUOR kit) (8), ALDH converts fluorescently-labelled aminoacetaldehyde, an ALDH substrate which freely diffuses into cells, into fluorescently-labelled aminoacetate, a negatively charged product that is retained inside cells. Therefore, cells with high ALDH activity accumulate increasing amounts of the fluorescent aminoacetate and are subsequently detected as ALDH-bright $\left(\mathrm{ALDH}^{\mathrm{br}}\right.$ ) cells by flow cytometry (8). ALDH is considered to be a detoxifying enzyme that protects stem cells from cytotoxic effects, and its activity is reported to be high in primitive hematopoietic stem cells $(8,9)$. Therefore, ALDH activity is considered to be a marker of stem cell activity. In peripheral blood stem cell (PBSC) transplantation, the number of ALDH ${ }^{\text {br }}$ cells in mobilized PBSC correlates with engraftment success following autologous transplantation (10). In UCBT, ALDH ${ }^{\text {br }}$ cells are correlated with CFU-GM, suggesting that ALDH activity may be suitable for quality assessment of CBUs (11).

In the present study, the association between TNCs, $\mathrm{ALDH}^{\mathrm{br}}$ cells, CD $34^{+}$cells and CFUs was examined in 16 CBUs. In addition, the number of $\mathrm{ALDH}^{\mathrm{br}}$ cells in the cryopreserved main bag were compared with those in the attached segment. The present results suggested that evaluation of ALDH activity 
in the cryopreserved CB cells of the attached segments may be useful for quality assessment prior to UCBT.

\section{Materials and methods}

CB collection and processing. Umbilical CB was collected into a $200 \mathrm{ml}$ bag containing $28 \mathrm{ml}$ citrate phosphate dextrose-adenine anticoagulant by venipuncture of the umbilical cord following full-term delivery, in cooperation with the Obstetric Clinic with Hyogo Cord Blood Bank (Nishinomiya, Japan). Informed consent was obtained from the mother to donate to the Cord Blood Unit (CBU) following $\mathrm{CB}$ transplantation or for medical research if not suitable for transplantation. CBUs that were not suitable for transplantation were selected for this study between November 2012 and December 2014. CB was mixed with 6\% hydroxyethyl starch (Nipro Pharma Corporation, Osaka, Japan) at a ratio of 5:1 for $5 \mathrm{~min}$, and centrifuged at $50 \mathrm{x}$ g for $5 \mathrm{~min}$ at room temperature. The buffy coat layer was subsequently transferred to a new bag. The buffy coat was cryopreserved in 5\% dimethylsulfoxide, 6\% hydroxyethyl starch (Kyokuto Pharmaceutical Industrial Co., Ltd., Tokyo, Japan) and 4\% human serum albumin (Mitsubishi Tanabe Pharma Corporation, Osaka, Japan) in a final volume of $25 \mathrm{ml}$ in the main bag, and $\sim 0.4 \mathrm{ml}$ in the attached tube which was divided into four attached segments (each volume was $\sim 0.125,0.055,0.11$ and $0.11 \mathrm{ml}$ ) by heat sealing. CBUs were cryopreserved at $-80^{\circ} \mathrm{C}$ without rate-controlled freezing overnight (12), and were subsequently transferred and stored in liquid nitrogen. For quality assessment, main bags and attached segments were thawed in a $37^{\circ} \mathrm{C}$ water bath, and $\mathrm{TNC}$ and $\mathrm{CD} 34^{+}$cell quantification, $\mathrm{CFU}$ assay and ALDH analysis were performed subsequently. The present study was approved by the institutional review board of Hyogo College of Medicine and Hyogo Cord Blood Bank (Nishinomiya, Japan).

Quantification of TNC and CD34 $4^{+}$cells. Quantification of TNCs was determined using an automated cell counter (XE-5000; Sysmex Corporation, Kobe, Japan). Quantification of $\mathrm{CD} 4^{+}$hematopoietic stem and progenitor cells was determined using a BD Stem Cell Enumeration kit (cat. no. 344563; BD Biosciences, San Jose, CA, USA), which included a fluorescein isothiocyanate-conjugated anti-CD45 monoclonal antibody (clone 2D1), a phycoerythrin-conjugated anti-CD34 antibody (clone 8G12) and 7-Amino-actinomycin D (7AAD). Cells at $2-20 \times 10^{6} / \mathrm{ml}$ were incubated with these antibodies and 7AAD for $20 \mathrm{~min}$ at room temperature in the dark, then erythrocytes were lysed with ammonium chloride solution. Samples were analyzed using a FACSCanto ${ }^{\mathrm{TM}}$ II system and BD FACSCanto ${ }^{\mathrm{TM}}$ Clinical software version 2.4 (BD Biosciences). $\mathrm{CD}_{3} 4^{+}$cells were identified under sequential gating of the $7 \mathrm{AAD}^{-}$and $\mathrm{CD} 45^{\mathrm{dim}+}$ cell populations according to the single platform guidelines of the International Society of Hematotherapy and Graft Engineering (13).

CFU assay. CFU assays were performed using a commercially available methylcellulose medium (MethoCult H4034 Optimum; Stemcell Technologies, Inc., Vancouver, $\mathrm{BC}$, Canada) according to the manufacturer's protocol. Briefly, 2-4x $10^{4} \mathrm{CB}$ cells were cultured in semisolid methylcellulose medium containing recombinant human (rh) stem cell factor, rh granulocyte-macrophage colony stimulating factor (GM-CSF), rh G-CSF and rh interleukin-3 and rh erythropoietin in $35-\mathrm{mm}$ petri dishes for 14 days at $37^{\circ} \mathrm{C}$ in a humidified atmosphere of $5 \% \mathrm{CO}_{2}$. CFU-GM, CFU-granulocyte/erythroid/macrophage/megakaryocyte (GEMM) and burst-forming units-erythroid (BFU-E) were identified by observation of GM, GEMM and erythroid colonies, respectively, using an inverted microscope as described previously (14). The number of each CFU was calculated as the mean number of colonies in three dishes. Total CFUs were calculated by summation of mean CFU-GM, BFU-E and CFU-GEMM values.

ALDH analysis. The ALDEFLUOR kit(Stemcell Technologies, Inc.) was used for detection of intracellular ALDH enzyme activity by flow cytometry, according to the manufacturer's protocol. Briefly, boron-dipyrromethene (BODIPY) fluorescent dye-labeled aminoacetaldehyde (BAAA) is a substrate for ALDH that diffuses freely into cells. This is converted by ALDH into BODIPY fluorescent dye-labeled aminoacetate (BAA), which remains trapped intracellularly, thereby emitting a green fluorescence that enables flow cytometric detection of $\mathrm{ALDH}^{\text {br }}$ cells (8). As a negative control, the background fluorescence of the $\mathrm{ALDH}^{\mathrm{br}}$ cells was obtained by inhibition of ALDH activity by diethylaminobenzaldehyde. Analysis of $\mathrm{ALDH}^{\text {br }}$ cells was performed using a FACSCanto II system.

Statistical analysis. Data are expressed as the mean \pm standard deviation or as the median (range). Paired t-tests were used to assess differences between the main bag and the attached segment. Linear regression analysis was performed to assess the correlation between the two groups. All statistical analyses were performed using GraphPad Prism version 6.0 for Windows (GraphPad Software, Inc., La Jolla, CA, USA). P $<0.05$ was considered to indicate a statistically significant difference.

\section{Results}

Characteristics of thawed cord blood units. In UCBT, CD34 ${ }^{+}$ cell counts and CFU counts in the CB graft are known to be good predictors for engraftment and patient survival. Therefore, in the present study, $\mathrm{ALDH}^{\mathrm{br}}$ cell counts for UBC quality assessment were evaluated by comparison with these parameters. The number of TNCs, $\mathrm{CD} 34^{+}$, and $\mathrm{ALDH}^{\mathrm{br}}$ cells and total CFUs were analyzed in the CB from the main bags of 16 CBUs, and detailed results are provided in Table I. The number of TNCs and $\mathrm{ALDH}^{\text {br }}$ cells were $8.06 \pm 1.70 \times 10^{8} / \mathrm{CBU}$ and $2.61 \pm 2.78 \times 10^{5} / \mathrm{CBU}$, respectively.

Association between $A L D H^{b r}$ cell count and $\mathrm{CD}^{2} 4^{+}$cells and total CFU counts. The association between TNCs, $\mathrm{ALDH}^{\mathrm{br}}$ cells, CD34 ${ }^{+}$cells and CFUs was subsequently examined by linear regression analysis. The number of TNCs were not significantly correlated with the number of $\mathrm{CD} 34^{+}$cells (Pearson's correlation coefficient $\mathrm{R}=0.1458, \mathrm{P}>0.05$ ) or the number of $\mathrm{ALDH}^{\mathrm{br}}$ cells ( $R=0.07254, P>0.05$; data not shown). As presented in Fig. 1, the number of $\mathrm{ALDH}^{\mathrm{br}}$ cells were significantly correlated with $\mathrm{CD} 34^{+}$cell counts $(\mathrm{R}=0.8686, \mathrm{P}<0.0001)$ in post-thaw $\mathrm{CB}$ in the main bag. Next, three different colony-forming 
Table I. Characteristics of thawed cord blood units in the main bags.

\begin{tabular}{|c|c|c|c|}
\hline Variables & Mean \pm SD & Median & Range \\
\hline TNCs (x $10^{8} /$ unit) & $8.06 \pm 1.70$ & 7.76 & $5.50-12.25$ \\
\hline $\mathrm{CD} 34^{+}$cells (x105/unit) & $13.77 \pm 8.91$ & 9.33 & $2.06-35.00$ \\
\hline $\mathrm{ALDH}^{\mathrm{br}}$ cells (x10\%/unit) & $2.61 \pm 2.78$ & 1.16 & $0.29-9.17$ \\
\hline CFU-GM (x105/unit) & $4.85 \pm 2.99$ & 4.24 & $0.72-12.42$ \\
\hline BFU-E (x105/unit) & $3.18 \pm 1.98$ & 3.37 & $0.72-7.69$ \\
\hline CFU-GEMM (x105/unit) & $0.04 \pm 0.14$ & 0.14 & $0-0.54$ \\
\hline Total CFUs (x105/unit) & $8.70 \pm 4.56$ & 6.82 & $1.44-17.46$ \\
\hline
\end{tabular}

Cryopreserved cord blood units in the main bags were thawed and analyzed $(n=16)$. TNCs, total nucleated cells; ALDH ${ }^{\text {br }}$, aldehyde dehydrogenase-bright; CFU-GM, colony forming units-granulocyte/macrophage; BFU-E, burst-forming units-erythroid; GEMM, granulocyte/erythroid/macrophage/megakaryocyte; SD, standard variation; CD34, cluster of differentiation 34.

unit assays were performed for myeloid lineage (CFU-GM), erythroid lineage (BFU-E) and mixed myeloid and erythroid lineage cells (CFU-GEMM). The association between ALDH ${ }^{\text {br }}$ cell counts and CFU-GM, BFU-E or total CFUs (as the sum of the CFU-GM, BFU-E and CFU-GEMM assays) in thawed CB in the main bag was examined. The number of $\mathrm{ALDH}^{\mathrm{br}}$ cells correlated with the number of CFU-GM $(\mathrm{R}=0.6608, \mathrm{P}=0.0053$; Fig. 2A) and BFU-E ( $R=0.6964, \mathrm{P}=0.0027$; Fig. 2B). Notably, the number of $\mathrm{ALDH}^{\mathrm{br}}$ cells exhibited a stronger correlation to the number of total CFUs ( $\mathrm{R}=0.7502, \mathrm{P}=0.0008$; Fig. 2C). Additionally, the number of total CFUs was positively correlated with the number of $\mathrm{CD} 34^{+}$cells $(\mathrm{R}=0.7224, \mathrm{P}=0.0016$; Fig. 2D). The significant correlation of $\mathrm{ALDH}^{\text {br }}$ cell count with $\mathrm{CD}^{+}{ }^{+}$cell and total CFU counts supports the hypothesis that measuring $\mathrm{ALDH}^{\mathrm{br}}$ cell numbers may serve as an indicator of CB quality following cryopreservation.

Comparison of ALDH ${ }^{\text {br }}$ cells in the main bag and the attached segment. The concentration of $\mathrm{ALDH}^{\text {br }}$ cells was subsequently evaluated in the main bags and attached segments of the CBUs. As presented Fig. 3A, the concentration of $\mathrm{ALDH}^{\mathrm{br}}$ cells in the main bag and in the attached segment was $10.05 \pm 1.76$ and $9.06 \pm 1.76$ cells $/ \mu 1$ of $C B$, respectively, which was not significantly different $(\mathrm{P}=0.1731)$. Furthermore, the concentration of $\mathrm{ALDH}^{\mathrm{br}}$ cells exhibited a high linear correlation between the main bags and attached segments in the 16 CBUs tested in the present study $(\mathrm{R}=0.9399, \mathrm{P}<0.0001$; Fig. 3B). These results suggested that $\mathrm{ALDH}^{\mathrm{br}}$ cell counts in the cryopreserved attached segments are similar to the main bag, and therefore may successfully serve as an indicator in CB quality assessment.

\section{Discussion}

Umbilical CB cells are an alternative source of hematopoietic stem cells for transplantation. TNC, CD $34^{+}$cell and CFU counts in a CBU have been previously demonstrated to positively correlate with good engraftment rate and improved survival following CBT (3); therefore, these parameters have been adopted as essential criteria for assessing UCB quality prior to transplantation. In the present study, ALDH activity in the $\mathrm{CB}$ was examined in parallel with $\mathrm{TNC}, \mathrm{CD} 34^{+}$cell and $\mathrm{CFU}$ counts, in order to evaluate it as a novel marker for UCB quality.

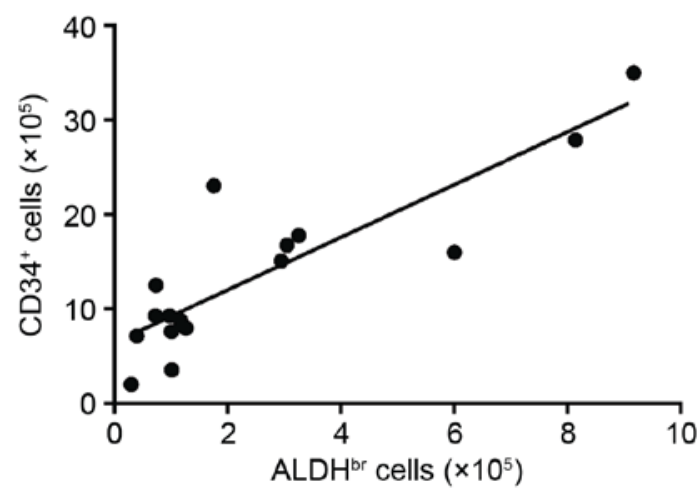

Figure 1. Correlation between the number of $\mathrm{ALDH}^{\mathrm{br}}$ cells and CD34+ cells in thawed cord blood in the main bag. Scatter plot of results from 16 cord blood units. Pearson's correlation coefficient $\mathrm{R}=0.8686, \mathrm{P}<0.0001 . \mathrm{ALDH}^{\mathrm{br}}$, aldehyde dehydrogenase-bright; CD34, cluster of differentiation 34.

ALDH oxidizes intracellular aldehydes to carboxylic acid, and this process is involved in cellular detoxification, proliferation, differentiation and drug resistance (10). ALDH ${ }^{\text {br }}$ cell presence is a novel marker for human hematopoietic stem cells (8). In the present study, the number of $\mathrm{ALDH}^{\mathrm{br}}$ cells was highly correlated with the number of $\mathrm{CD} 34^{+}$cells. Storms et al (9) reported that the $\mathrm{ALDH}^{\mathrm{br}} / \mathrm{CD} 34^{+}$cell population contains primitive hematopoietic stem cells and progenitor cells. The same study addiitonally reported that $\mathrm{ALDH}^{-} / \mathrm{CD}^{+} 4^{+}$ cells do not contain primitive stem cells but contain a few progenitor cells, while $\mathrm{ALDH}^{\mathrm{br}} / \mathrm{CD} 34^{-}$cells exhibit no hematopoietic activity but contain erythroid cells (9). Thus, ALHD ${ }^{\text {br }}$ cells represent primitive hematopoietic stem and committed progenitor cells. The results of the present study suggested that $\mathrm{ALDH}^{\mathrm{br}}$ cells may serve as a novel indicator of CB quality; however, whether $\mathrm{ALDH}^{\text {br }}$ cells are more reliable indicators than $\mathrm{CD}_{3} 4^{+}$cells requires further clarification.

Lee et al (11) reported that $\mathrm{ALDH}^{\text {br }}$ cells are correlated with post-thaw CFU-GM counts in CB. The present study supported this finding and further demonstrated that $\mathrm{ALDH}^{\mathrm{br}}$ cells were correlated with BFU-E and total CFU counts. In particular, total CFU counts exhibited the strongest correlation coefficient among CFUs. Previous reports have used either CFU-GM (11) or total CFU (4) counts for assessment 
A

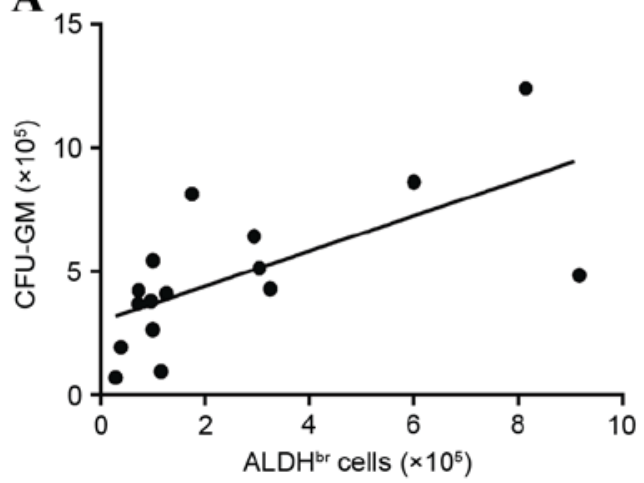

C

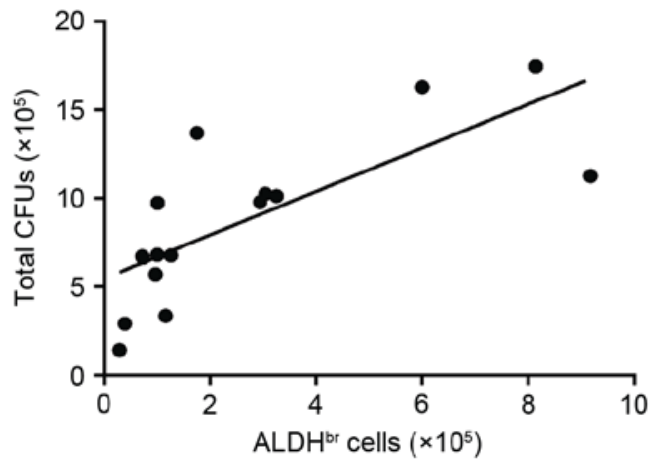

B

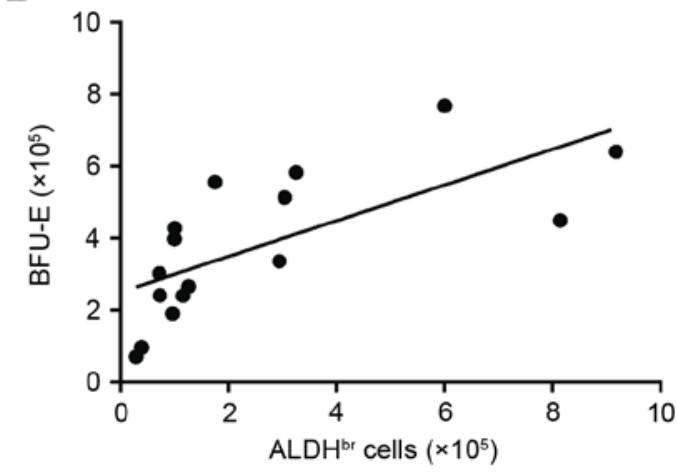

D

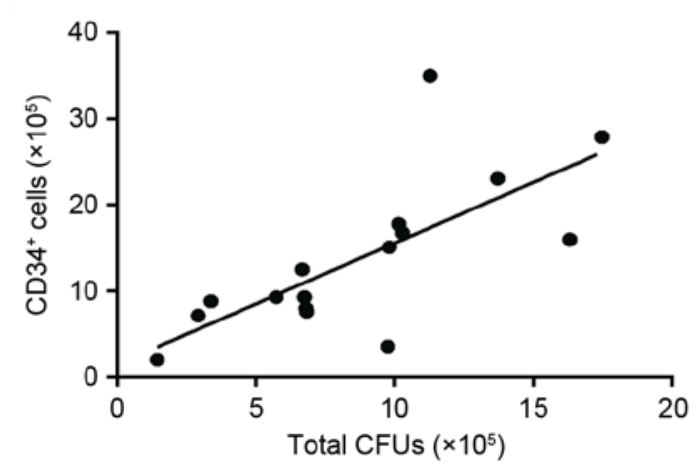

Figure 2. Correlation among the numbers of $\mathrm{ALDH}^{\mathrm{br}}$ cells, $\mathrm{CD} 34^{+}$cells and CFUs in thawed cord blood cells in the main bag. Scatter plots of ALDH ${ }^{\text {br }}$ cell numbers and (A) CFU-GM (Pearson's correlation coefficient $R=0.6608, P=0.0053$ ), (B) BFU-E ( $R=0.6964, P=0.0027$ ), and (C) total $C F U$ numbers $(R=0.7502$, P.0008). (D) Scatter plot of total CFU and $\mathrm{CD} 34^{+}$cell numbers $(\mathrm{R}=0.7224, \mathrm{P}=0.0016)$. Results represent analysis of 16 cord blood units. ALDH ${ }^{\mathrm{br}}$, aldehyde dehydrogenase-bright; CFU, colony forming unit; GM, granulocyte/macrophage; BFU-E, burst-forming units-erythroid; CD34, cluster of differentiation 34 .
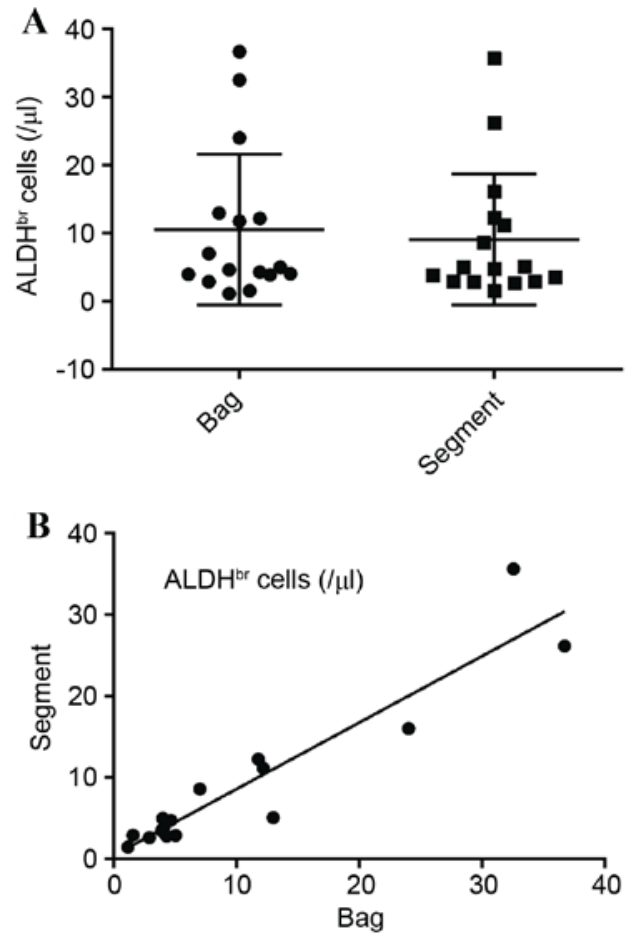

Figure 3. Comparison of $\mathrm{ALDH}^{\mathrm{br}}$ cells in the main bag and the attached segment. (A) Concentration of $\mathrm{ALDH}^{\mathrm{br}}$ cells per $\mu 1$ of cord blood in the main bag and the attached segment $(\mathrm{P}=0.1731$, not significant). Data are presented as the mean \pm standard deviation. (B) Scatter plot of $\mathrm{ALDH}^{\text {br }}$ cell concentrations in the main bag and the attached segment (Pearson's correlation coefficient $\mathrm{R}=0.9399, \mathrm{P}<0.0001)$. Results represent analysis of 16 cord blood units. ALDH ${ }^{\text {br }}$, aldehyde dehydrogenase-bright. of hematopoietic activity in transplantation. The quality and quantity of mobilized PBSCs he been assessed by CFU-GM counts (15). In unpublished results from our group, CFU assay of mobilized PBSCs gave rise to $>90 \%$ of CFU-GM colonies and only a few BFU-E and CFU-GEMM colonies (data not shown). This finding may explain why CFU-GM is commonly used for quality assessment of mobilized PBSCs in PBSC transplantation. By contrast, $\mathrm{CB}$ cells gave rise to approximately $40 \%$ of CFU-GM, 50\% of BFU-E and 10\% of CFU-GEMM colonies (data not shown). Therefore, CFU-GM represent $<50 \%$ total CFUs and the proportion of CFU-GM and non-CFU-GM (i.e., the sum of BFU-E and CFU-GEMM) differ considerably among individuals, indicating that CFU-GM may not be a suitable indicator of CFUs. Accordingly, in the present study, the number of ALDH ${ }^{\text {br }}$ cells was better correlated with total CFUs than CFU-GM. Thus, total CFUs may be a better marker than CFU-GM in the quality assessment of CB.

Attached segments of CBUs are routinely used for quality assessment of the unit selected for transplantation. Rodríguez et al (7) demonstrated that there are no differences in the calculated number of TNCs, $\mathrm{CD} 34^{+}$cells and CFUs between the main bag and the attached segment; thus, the attached segment is considered to be representative of the $\mathrm{CBU}$ in the main bag. However, another report demonstrated that the attached segment exhibited higher numbers of $\mathrm{CD} 34^{+}$ cells and CFU-GM than the main bag (16). Furthermore, the attached segment is much smaller in volume compared with the main bag, and extra caution is required in handling the attached segment to avoid a rise in temperature that may be 
harmful to CB cells. Therefore, a comparison of ALDH ${ }^{\text {br }}$ cell concentration in the main bag and the attached segment was performed in the present study, to ascertain the suitability of the attached segment as representative for the main bag. The results demonstrated that the concentration of $\mathrm{ALDH}^{\mathrm{br}}$ cells in the attached segment was comparable to that in the main bag.

In conclusion, the present study examined the use of ALDH activity in the attached segments as a novel indicator of UBC quality assessment in the main bag prior to transplantation. Shoulars et al (17) have recently reported similar results that ALHD $^{\text {br }}$ cells in the segment are associated with CFUs and may be utilized for quality assays prior to shipping from a cord blood bank. Larger clinical studies will be required in the future to examine the correlation between the number of $\mathrm{ALDH}^{\mathrm{br}}$ cells and patient survival following UCBT.

\section{Acknowledgements}

The authors would like to thank Mrs Mayuko Ohno and Mrs. Tika Tanihara (Hyogo Cord Blood Bank, Nishinomiya, Japan) for their technical assistance. The authors would also like to thank Mr. Kazuya Ashida (Hyogo Cord Blood Bank, Nishinomiya, Japan) for the technical advice in the preparation of this manuscript.

\section{Funding}

The present study was supported by the research grant from the Hyogo College of Medicine (grant received by YF).

\section{Availability of data and materials}

The analyzed datasets generated during the study are available from the corresponding author on reasonable request.

\section{Authors' contributions}

JI and TK performed the experiments using umbilical cord blood cells. SY analyzed and interpreted the data. SK and TK contributed for collecting and processing of umbilical cord blood. YF conceived and design the study and was a major contributor in writing the manuscript. All authors read and approved the final manuscript.

\section{Ethics approval and consent to participate}

The study was approved by the institutional review board of Hyogo College of Medicine and Hyogo Cord Blood Bank (Nishinomiya, Japan). Informed consent was obtained from the mother to donate cord blood to Hyogo Cord Blood Bank for CB transplantation or for medical research if not suitable for transplantation.

\section{Patient consent for publication}

Not applicable.

\section{Competing interests}

The authors declare that they have no competing interests.

\section{References}

1. Gluckman E, Rocha V, Boyer-Chammard A, Locatelli F, Arcese W, Pasquini R, Ortega J, Souillet G, Ferreira E, Laporte JP, et al: Outcome of cord-blood transplantation from related and unrelated donors. Eurocord Transplant Group and the European Blood and Marrow Transplantation Group. N Engl J Med 337: 373-381, 1997.

2. Laughlin MJ, Eapen M, Rubinstein P, Wagner JE, Zhang MJ Champlin RE, Stevens C, Barker JN, Gale RP, Lazarus HM, et al: Outcomes after transplantation of cord blood or bone marrow from unrelated donors in adults with leukemia. N Engl J Med 351: 2265-2275, 2004.

3. Wagner JE, Barker JN, DeFor TE, Baker KS, Blazar BR, Eide C, Goldman A, Kersey J, Krivit W, MacMillan ML, et al: Transplantation of unrelated donor umbilical cord blood in 102 patients with malignant and nonmalignant diseases: Influence of CD34 cell dose and HLA disparity on treatment-related mortality and survival. Blood 100: 1611-1618, 2002.

4. Page KM, Zhang L, Mendizabal A, Wease S, Carter S, Gentry T, Balber AE and Kurtzberg J: Total colony-forming units are a strong, independent predictor of neutrophil and platelet engraftment after unrelated umbilical cord blood transplantation: A single-center analysis of 435 cord blood transplants. Biol Blood Marrow Transplant 17: 1362-1374, 2011.

5. Broxmeyer HE, Srour EF, Hangoc G, Cooper S, Anderson SA and Bodine DM: High-efficiency recovery of functional hematopoietic progenitor and stem cells from human cord blood cryopreserved for 15 years. Proc Natl Acad Sci USA 100: 645-650, 2003.

6. Kudo Y, Minegishi M, Seki O, Takahashi H, Suzuki A, Narita A, Sato Y, Abe M, Ishioka N, Harigae H and Tsuchiya S: Quality assessment of umbilical cord blood units at the time of transplantation. Int J Hematol 93: 645-651, 2011.

7. Rodríguez L, García J and Querol S: Predictive utility of the attached segment in the quality control of a cord blood graft. Biol Blood Marrow Transplant 11: 247-251, 2005.

8. Storms RW, Trujillo AP, Springer JB, Shah L, Colvin OM, Ludeman SM and Smith C: Isolation of primitive human hematopoietic progenitors on the basis of aldehyde dehydrogenase activity. Proc Natl Acad Sci USA 96: 9118-9123, 1999.

9. Storms RW, Green PD, Safford KM, Niedzwiecki D, Cogle CR, Colvin OM, Chao NJ, Rice HE and Smith CA: Distinct hematopoietic progenitor compartments are delineated by the expression of aldehyde dehydrogenase and CD34. Blood 106: 95-102, 2005.

10. Fallon P, Gentry T, Balber AE, Boulware D, Janssen WE, Smilee R, Storms RW and Smith C: Mobilized peripheral blood SSCloALDHbr cells have the phenotypic and functional properties of primitive haematopoietic cells and their number correlates with engraftment following autologous transplantation. Br J Haematol 122: 99-108, 2003.

11. Lee HR, Shin S, Yoon JH, Roh EY, Kim BJ and Song EY: Aldehyde dehydrogenase-bright cells correlated with the colony-forming unit-granulocyte-macrophage assay of thawed cord blood units. Transfusion 54: 1871-1875, 2014.

12. Makino S, Harada M, Akashi K, Taniguchi S, Shibuya T, Inaba S and Niho Y: A simplified method for cryopreservation of peripheral blood stem cells at -80 degrees $C$ without rate-controlled freezing. Bone Marrow Transplant 8: 239-244, 1991.

13. Keeney M, Chin-Yee I, Weir K, Popma J,Nayar R and Sutherland DR: Single platform flow cytometric absolute $\mathrm{CD} 34^{+}$cell counts based on the ISHAGE guidelines. International Society of Hematotherapy and Graft Engineering. Cytometry 34: 61-70, 1998.

14. Fujimori Y, Hara $\mathrm{H}$ and Nagai K: Effect of lymphokine-activated killer cell fraction on the development of human hematopoietic progenitor cells. Cancer Res 48: 534-538, 1988.

15. Fu SQ, Abboud CN, Brennan JK, Ifthikharuddin JJ, Nichols D and Liesveld JL: Impact of mobilized blood progenitor cell quality determined by the CFU-GM/CD34+ ratio on rapid engraftment after blood stem cell transplantation. Blood Cells Mol Dis 28: 315-321, 2002.

16. Lee HR, Shin S, Yoon JH, Roh EY, Song EY, Han KS and Kim BJ: Attached segment has higher CD34+ cells and CFU-GM than the main bag after thawing. Cell Transplant 24: 305-310, 2015.

17. Shoulars K, Noldner P, Troy JD, Cheatham L, Parrish A, Page K, Gentry T, Balber AE and Kurtzberg J: Development and validation of a rapid, aldehyde dehydrogenase bright-based cord blood potency assay. Blood 127: 2346-2354, 2016. 